\section{P72 LIVED EXPERIENCES OF MIDWIVES AND TRADITIONAL BIRTH ATTENDANTS CARING FOR PREGNANT TEENAGERS AND TEENAGE MOTHERS: A PHENOMENOLOGICAL STUDY}

1,2S Gbogbo*, ${ }^{1} \mathrm{M}$ Ayanore, ${ }^{3} Y$ Enuameh, ${ }^{2} \mathrm{C}$ Schweppe. ${ }^{1}$ School of Public Health, University of Health and Allied Sciences, Ho, Ghana; ${ }^{2}$ Institute of Education, Johannes Gutenberg University, Mainz, Germany; ${ }^{3}$ School of Public Health, Kwame Nkrumah University of Science and Technology, Kumasi, Ghana

\subsection{6/jech-2020-SSMabstracts. 164}

Background Teenage mothers are confronted with the double task of passing through the teenage phase and, at the same time, familiarizing themselves with the requirements of motherhood. The characteristics connected with the teenage stage mostly affect the capability of teenage mothers to adapt to the role of being a first-time mother. The essential role of midwives is to provide care for women during pregnancy and motherhood, through coaching, reassurance, supervision and social support. Traditional Birth Attendants (TBAs) are also valued as essential actors in maternal health, providing social support and other antenatal, intrapartum and postnatal care in developing countries. The study aimed to explore a deeper understanding of the lived experiences of midwives and traditional birth attendants in caring for pregnant teenagers and teenage mothers.

Methods A descriptive phenomenological framework based on a lifeworld approach was used, with in-depth interviews as the method of data collection. The setting of the study was a municipal hospital and the seven districts in the Hohoe municipality, Ghana. Six midwives and six TBAs aged 37 to 59, with experience in caring for pregnant teenagers and teenage mothers, were interviewed. Data collected from the midwives and the TBAs was analyzed using Giorgi's data analysis process.

Results The general structure of the phenomenon can be described in two main themes with four sub-themes. The first main theme, 'The midwife and the traditional birth attendant as being the mother figure', included 'Emotional and financial support', and 'Creating trust and rapport'. The second main theme, 'The frightened teenage woman', included 'Unprepared: not ready for pregnancy and motherhood' and 'Unsupported: relationship with parents'.

Conclusion The midwives and traditional birth attendants played the role of being a mother to these teenagers by creating trust and supporting them financially. The teenagers were not prepared for pregnancy and motherhood, and that some parents did not create good relationships with their daughters. The study has the potential to increase knowledge and understanding of midwives and TBAs' lived experience of caring for teenagers during pregnancy and early motherhood and therefore has implications for practice, education, and research.

\section{P73 TRENDS, WEALTH INEQUALITIES AND THE ROLE OF THE PRIVATE SECTOR IN CAESAREAN SECTION IN THE ARAB REGION: A REPEAT CROSS-SECTIONAL ANALYSIS OF POPULATION-BASED SURVEYS}

${ }^{1} \mathrm{SJ} \mathrm{McCall}{ }^{*},{ }^{2} \mathrm{~N}$ Altijani, ${ }^{3} \mathrm{~T}$ Kabakian-Khasholian. ${ }^{1}$ Center for Research on Population and Health, Faculty of Health Sciences, American University of Beirut, Beirut, Lebanon; ${ }^{2}$ National Perinatal Epidemiology Unit, University of Oxford, Oxford, UK; ${ }^{3}$ Health Promotion and Community Health, Faculty of Health Sciences, American University of Beirut, Beirut, Lebanon

10.1136/jech-2020-SSMabstracts. 165
Background The Arab region has some of the highest caesarean section rates globally; however, trends and inequalities have not been explored regionally. This study aimed to examine the trends of caesarean section and describe variations in caesarean section by economic status and type of healthcare facility (private/public sector).

Methods This was a secondary data analysis of the two most recent(2006-2018) demographic and health surveys(DHS) or multiple indicator cluster surveys(MICS) for eight countries in the Arab region (Algeria, Egypt, Iraq, Jordan, Qatar, Tunisia, State of Palestine and Yemen). DHS/MICS are nationally representative household surveys; the sampling frame includes area units across the entire country, and employs a multi-stage stratified cluster sampling procedure, to provide a sample population of women aged 15-49 years who had a live birth in the preceding two years. The outcome was caesarean section at last birth. Temporal trends were calculated using generalised linear models and presented as risk differences(RD) with 95\% confidence intervals $(95 \% \mathrm{CI})$. Caesarean section was disaggregated against household wealth index and type of healthcare facility (private/public sector) and presented using equiplots. Analyses accounted for the complex sampling design and were conducted using STATA(v.15).

Results In the most recent survey, use of caesarean section ranged from $57.3 \%$ of births (95\%CI:55.6-59.1\%) in Egypt to $5.7 \%$ of births (95\%CI:5.0-6.6\%) in Yemen. Overall, the use of caesarean section has increased across the Arab region with the exception of Jordan, which had no statistically significant change during 2012-2018 shown by an RD of $-3.0 \%$ (95\%CI:-7.4-1.4\%). Within all Arab countries, caesarean section use was highest in the richest quintile compared to the poorest quintile, for example in Iraq: $47.6 \%$ (95\%CI:41.4$54.0 \%)$ vs. $27.7 \%$ (95\%CI:24.5-31.1\%), respectively. Caesarean section use was higher in private sector facilities compared to public sector: $70.2 \%$ (95\%CI:68.2-72.1\%) vs. $50.8 \%$ (95\% CI:47.6-54.1\%) in Egypt, and 21.9\% (95\%CI:18.1-26.1\%) vs. 15.7\% (95\%CI:13.3-18.4\%) in Yemen, respectively. Excluding Egypt, there was a larger absolute number of births in public sector facilities compared to private sector facilities.

Conclusion Variations in the use of caesarean section exist within and between Arab countries, and it was unequally distributed amongst the richest quintiles and private healthcare facilities. The self-reported nature of the data is a notable limitation. The private sector has a prominent role in the observed trends; however, moderate increases in the use of caesarean section within the public sector will result in a larger absolute increase nationally. Urgent policies and interventions are required to address non-medically indicated caesarean sections.

\section{P74 BARRIERS AND FACILITATORS FOR PRECONCEPTION PLANNING AMONG WOMEN IN THE UNITED KINGDOM: A RAPID REVIEW}

${ }^{1} \mathrm{~A}$ Ayorinde, ${ }^{1} \mathrm{~F}$ Boardman, ${ }^{1} \mathrm{~B}$ Alzouebi, ${ }^{2} \mathrm{~L}$ Porter, ${ }^{3} \mathrm{~A}$ Hadley, ${ }^{3} \mathrm{M}$ Ludeke, ${ }^{2} \mathrm{~A}$ Sallis, ${ }^{3} S$ Mann, ${ }^{1} O$ Oyebode*. ${ }^{1}$ Warwick Medical School, University of Warwick, Coventry, UK; ${ }^{2}$ Behavioural Insights Team, Public Health England, London, UK; ${ }^{3}$ Programmes and Priorities, Public Health England, London, UK

\subsection{6/jech-2020-SSMabstracts. 166}

Background Preconception planning enables women to optimize preconception health thereby reducing the risk of poor maternal and neonatal outcomes. Awareness and practice of 
preconception planning is generally low. In this rapid evidence review, we examined the barriers and facilitators to women choosing to plan and prepare for a healthy pregnancy.

Methods We searched MEDLINE, PsycINFO and CINAHL from 2009 to October 2019 and limited to English language. We included any publications that presented facilitators and barriers for women choosing to plan and prepare for pregnancy. We also included papers presenting barriers and facilitators to health care professionals supporting this behaviour and articles on relevant interventions although these data are not presented here. We contacted experts to identify grey literature. We extracted study characteristics using a pre-piloted data extraction form and assessed the quality of individual studies using the Mixed Methods Appraisal Tool. One reviewer performed title and abstract screening, data extraction and quality assessment with a sample checked by a second reviewer. Two reviewers screened full texts independently. Using NVivo, we coded information on barriers and facilitators from each study into themes under two subheadings; a) information seeking and b) preparing for a healthy pregnancy.

Results We screened 2679 citations, 54 full-text articles and included 24 articles for analysis. 18articles reported barriers and facilitators for women. The most frequently reported barriers to information seeking prior to conception were unintended pregnancy, information provoking anxiety, and belief that there is no need for preconception care. Facilitators included ad hoc prompts in health care settings, and opportunities to discuss pregnancy intentions (e.g. as part of care for a chronic condition). The most frequent barriers to preparing for a healthy pregnancy were: not knowing what recommended behaviours might be, lack of understanding or incorrect beliefs, information not appropriate for woman's context, and lived experience (of self or in social circle) that appears to contradict health advice. Facilitators included knowledge of recommended behaviours, feelings of responsibility towards a potential baby, and confidence in ability to achieve health goals prior to conceiving.

Conclusion We will conduct a behavioural analysis and categorise the identified barriers and facilitators into the Theory and Techniques Tool (TaTT) mechanisms of actions (MoAs). We will then examine whether existing intervention content matches what is theoretically appropriate, therefore identifying opportunities for improvement of existing interventions and novel development to promote preconception planning and ultimately, better maternal and neonatal outcomes.

\section{P75 SYSTEMATIC REVIEW OF THE EFFECTIVENESS OF RIGHTS-BASED APPROACHES TO SEXUAL AND REPRODUCTIVE HEALTH IN LOW AND MIDDLE-INCOME COUNTRIES}

${ }^{1} \mathrm{M}$ McGranahan*, ${ }^{2} \mathrm{~J}$ Nakyeyune, ${ }^{2} \mathrm{C}$ Baguma, ${ }^{2} \mathrm{NN}$ Musisi, ${ }^{2} \mathrm{D}$ Nsibirwa, ${ }^{3} \mathrm{~S}$ Sekalala, ${ }^{1} O$ Oyebode. 'Warwick Medical School, University of Warwick, Coventry, UK; ${ }^{2}$ Center for Health, Human Rights and Development, Kampala, Uganda; ${ }^{3}$ School of Law, University of Warwick, Coventry, UK

\subsection{6/jech-2020-SSMabstracts. 167}

Background By 2030, the Sustainable Development Goals aim to achieve human rights for all. This involves empowering women and girls (Goal 5) and ensuring that everyone can access sexual and reproductive health rights (Goal 3). This is the first systematic review of the effectiveness of rights-based approaches to sexual and reproductive health including gender-based violence (GBV), maternity, HIV and sexually transmitted infections (STI).

Aim To determine the effectiveness of rights-based approaches to sexual and reproductive health in low and middle-income countries (LMICs).

Methods Search Strategy EMBASE, MEDLINE and Web of Science were searched until 9/1/2020. Inclusion criteria were:

- Study design: any interventional study

- Population: adolescent and adult females

- Setting: LMICs

- Intervention: a 'rights-based approach' (defined by the author) and/or interventions that the author explicitly stated related to 'rights'.

Study selection, data extraction and risk of bias assessment was undertaken independently by two authors.

Synthesis A narrative synthesis of included studies was undertaken, and outcomes mapped to identify evidence gaps.

Risk of bias The RoB-2 tool and the ROBINS-I tool were used to assess risk of bias of cluster-randomised studies and non-randomised studies respectively. Uncontrolled before-after studies based on two cross-sectional surveys were given an overall assessment of serious or critical risk of bias.

Results Of 17,212 records identified through database searching, 13,404 records remained after de-duplication. Sixty-nine studies remained following title and abstract screening, of which seven were included after full-text screening. Reference list screening identified seventeen studies.

Rights-based interventions were effective for most included outcomes, but evidence was of poor quality. Testing uptake for HIV and/or other STIs improved with intervention but all relevant studies were at critical or serious risk of bias. Condom use improved with intervention (although one study showed no change), but all relevant studies were at high, serious or critical risk of bias. Awareness of rights improved with intervention, but all four studies were at critical or serious risk of bias.

Conclusion This is the first systematic review to evaluate the effect of rights-based approaches to sexual and reproductive health including maternity, GBV and STIs/HIV. Considerable risk of bias in all studies means results must be interpreted with caution.

Priority and relevance Rights-based approaches are often recommended but high-quality controlled studies are needed urgently to determine if they are effective for sexual and reproductive health in LMICs.

This systematic review was written through a collaboration between the University of Warwick and the Center for Health Human Rights \& Development (CEHURD), Uganda.

\section{P76 THE ASSOCIATIONS BETWEEN PREGNANCY LOSS AND CARDIOVASCULAR DISEASES IN THE UK BIOBANK}

${ }^{1}$ FM Simmons-Jones*, ${ }^{2} \mathrm{C}$ Oliver-Williams. ${ }^{1}$ Public Health, Essex County Council, Chelmsford, UK: ${ }^{2}$ Cardiovascular Epidemiology Unit, Department of Public Health and Primary Care, University of Cambridge, Cambridge, UK

\subsection{6/jech-2020-SSMabstracts. 168}

Rationale This study assessed the associations between pregnancy loss (miscarriage, stillbirth and abortion) and future 